\title{
La actualidad de la antropología latinoamericana y caribeña: nuevas preguntas, nuevos desafíos teóricos y metodológicos
}

\author{
Laura $R$ Valladares' \\ Rebecca Lemos Igreja \\ 'Departamento de Antropología, Universidad Autónoma Metropolitana Iztapalapa, México \\ ${ }^{2}$ Universidade de Brasília, Departamento de Estudos Latino-americanos, \\ Programa de pós-graduação da Faculdade de Direito, Brasília/DF, Brasil
}

\section{Resumen}

Para este número de la revista Vibrant traemos las contribuciones de distintas.os antropólogas.os latinoamericanas.os y latinoamericanistas que reflexionan acerca de los grandes temas, problemáticas y abordajes teóricos metodológicos que ocupan actualmente a nuestra disciplina. Partimos de considerar que, si bien la antropología producida en América Latina y el Caribe se ha desarrollado de forma diversa, tiene la particularidad de ser una disciplina centrada en el análisis de los grandes problemas contemporáneos, sean los vinculados con la diversidad étnico cultural, las relaciones interculturales, las formas organizativas contrahegemónicas o con los impactos de la globalización, la diferencia y la desigualdad. Aunque no tengamos la pretensión de abarcar la multiplicidad de las antropologías latinoamericanas, buscamos destacar la diversidad de estudios, temas, dilemas y desafíos del quehacer antropológico en la contemporaneidad en la región y contribuir para el debate sobre la disciplina a lo cual tantas.os colegas se han dedicado.

Palabras clave: antropología; América Latina; Caribe; globalización; cultura. 


\title{
A atualidade da Antropologia latino- americana e caribenha: novas questões, novos desafios teóricos e metodológicos
}

\section{Resumo}

Para este número da revista Vibrant, trazemos as contribuições de diferentes antropólogas.os latinoamericanas.os e latino-americanistas que refletem sobre as grandes questões, problemas e abordagens teóricometodológicas que atualmente ocupam nossa disciplina. Começamos por considerar que, embora a antropologia produzida na América Latina e no Caribe tenha se desenvolvido de forma diversa, tem a particularidade de ser uma disciplina voltada para a análise dos grandes problemas contemporâneos, sejam eles relacionados à diversidade étnico-cultural, relações interculturais, formas de organização contra-hegemônicas ou com os impactos da globalização, a diferença e a desigualdade. Embora não pretendamos abarcar a multiplicidade das antropologias latino-americanas, buscamos destacar a diversidade de estudos, temas, dilemas e desafios do trabalho antropológico contemporâneo na região e contribuir para o debate sobre a disciplina a que tantos colegas se dedicam.

Palavras-chave: antropologia; América Latina; Caribe; globalização; cultura.

\section{Latin American and Caribbean Anthropology today: new questions, new theoretical and methodological challenges}

\begin{abstract}
For this issue of Vibrant, we bring the contributions of different Latin American anthropologists, or those dedicated to the study of the region, who reflect on the major issues, problems and theoretical methodological approaches that currently occupy our discipline. We start by considering that, although anthropology produced in Latin America and the Caribbean has developed in a diverse way, it has the particularity of being a discipline focused on the analysis of major contemporary problems, be they those related to ethniccultural diversity, intercultural relations, counter-hegemonic organizational forms or with the impacts of globalization, difference and inequality. Although we do not pretend to encompass the multiplicity of Latin American anthropologies, we seek to highlight the diversity of studies, themes, dilemmas, and challenges of contemporary anthropological work in the region and contribute to the debate on the discipline to which so many colleagues have been dedicated.
\end{abstract}

Keywords: anthropology; Latin America; Caribbean; globalization; culture. 


\title{
La actualidad de la antropología latinoamericana y caribeña: nuevas preguntas, nuevos desafíos teóricos y metodológicos
}

\author{
Laura $R$ Valladares \\ Rebecca Lemos Igreja
}

Para este número de la revista Vibrant convocamos a distintas.os antropólogas.os latinoamericanas.os y latinoamericanistas a reflexionar acerca de los grandes temas, problemáticas y abordajes teóricos metodológicos que ocupan actualmente a nuestra disciplina. Partimos de considerar que, si bien la antropología producida en América Latina y el Caribe se ha desarrollado de forma diversa y tiene una larga historia - pues en algunos países se remonta a más de un siglo, como en el caso de México, y en otros a medio siglo, como en Ecuador o Chile, en otros es más reciente - tiene la particularidad de ser una disciplina centrada en el análisis de los grandes problemas de nuestras naciones, sean los vinculados con la diversidad étnico cultural, las relaciones interculturales, las formas organizativas contrahegemónicas o con los impactos de la globalización, la diferencia y la desigualdad que recorren el mundo contemporáneo y tienen sus especificidades en nuestro continente. A este vínculo entre antropología y estudios de nuestras naciones, desde nuestra pertenencia ciudadana a las sociedades que estudiamos, Myriam Jimeno la ha denominado antropología ciudadana (Jimeno y Arias, 2011), es decir, se trata de una disciplina preocupada por estudiar nuestra propia diversidad y nuestras otredades, en el contexto de la globalización. En cada país la antropología se desarrolló de una manera particular, asumiendo diferentes paradigmas teóricos y respondiendo a contextos nacionales específicos y que en buena medida expresan el lugar que ocupa y ha ocupado el conocimiento social y humanista en distintos momentos históricos. Por ello consideramos que es pertinente hablar de antropologías latinoamericanas y caribeñas en plural.

Vivimos tiempos convulsos y de grandes trasformaciones en América Latina y nuevos temas han convocado a los antropólogos en las últimas décadas. Problemáticas como las racializaciones e intolerancias frente a la diversidad, los retos de la interculturalidad y fundamentalismos identitarios, así como los tránsitos de modelos políticos progresistas a los giros hacia la derecha son algunos de los nodos analíticos de nuestra disciplina. La xenofobia se extiende en momentos en que las migraciones Sur-Norte se han incrementado y los desplazamientos de grandes contingentes son detenidos con la construcción de muros. A contrapelo surgen movimientos de reivindicación de reconocimiento étnico-cultural y de derechos colectivos, como es el caso de los afrodescendientes por todo el continente y de los pueblos indígenas que se defienden, principalmente, de los despojos que los megaproyectos causan. Nuevos conceptos y problemáticas emergen para comprender y denunciar los grandes desastres sociales, ecológicos y políticos en esta era denominada como del antropoceno, para dar cuenta del rol de la actividad humana en la devastación ecológica del planeta. Recuperando la noción de crisis civilizatoria del filósofo lacaniano Slavoj Žižek distintas.os especialistas (Bartra, 2021; Leyva, et al. 2015) dan cuenta del momento político social contemporáneo y afirman que estamos ante una crisis multidimensional que afecta todas las áreas de la vida. Es una crisis del modelo de producción y consumo que se torna insostenible, que amenaza la vida del planeta, y que para algunos significa la fase terminal del patrón civilizatorio de la modernidad occidental capitalista. La crisis ambiental y el cambio climático son sólo una de sus más graves y 
evidentes manifestaciones. Por ello, en este dossier consideramos de enorme importancia hacer un recuento de los senderos que recorre la antropología, no solo para dar cuenta de los conflictos y dilemas del mundo social, sino también para pensar en nuevos horizontes.

En este escenario complejo y desconcertante este número de la revista inicia con el sugerente artículo de Néstor García Canclini ¿Qué será la interculturalidad?, en el que se propone reimaginar las tareas actuales de la antropología revisando cómo trataron los malentendidos interculturales antropólogas.os y sociólogas.os de varios países como son Marc Abélès, Roberto da Matta, Gustavo Lins Ribeiro, Claudio Lomnitz, Guillermo O’Donnell, Renato Ortiz, Rossana Reguillo y Ana Rosas Mantecón, así como los estudios recientes sobre jóvenes, tecnologías e intercambios académicos. Canclini reflexiona sobre el papel de las discrepancias en la interacción entre culturas en un tiempo en el que ganan fuerza los fundamentalismos identitarios. Propone hablar de interculturalidad agresiva para reconocer los conflictos del siglo XXI, en los cuales la coexistencia ineludible se vuelve insoportable y los deseos de alejar a los diferentes y quitarles derechos se hacen presentes. Las migraciones masivas y las industrias evidencian que los desentendimientos y disputas se multiplican internacional e intercontinentalmente. Con el confinamiento impuesto globalmente por la pandemia de COVID-19 y con las pérdidas económicas acentuando la aprensión hacia lo distinto, en estas circunstancias, las antropologías de lo local y nacional se quedan cortas para contribuir con la comprensión del contexto. El autor afirma que repensar la cultura y la ciudadanía en una época transnacional es desafiado. Por esta razón, nos propone hablar de una interculturalidad politeísta, la cual encuentra ser un recurso atractivo para fundar políticas de coexistencia ciudadana plural, aunque reconoce que se vuelve problemática al llegar, en tantos países, ante el exasperado fundamentalismo reciente. Lastimosamente, el autoritarismo y la militarización, legitimados por vías electorales, plantean problemas inéditos a la sobrevivencia democrática y a la convivencia intercultural. Son fenómenos importantes de analizar para repensar no solo nuestra disciplina, sino los modelos culturales y de gestión de la diversidad.

Sobre estos desencantos algunas.os antropólogas.os han dedicado sus pesquisas, muestra de ello son los siguientes cuatro artículos de este dossier, que fueron preparados por Rebecca Lemos Igreja, Eduardo Restrepo, Laura Valladares y Cristina Oehmichen. El artículo de Rebecca Lemos Igreja, intitulado Populism, inequality, and the construction of the "other": an anthropological approach to the far right in Brazil, coloca en el centro de su reflexión las implicaciones de los giros políticos hacia la extrema derecha. Presenta una síntesis muy pertinente centrada en recuperar las discusiones y aportes de las perspectivas antropológicas en el estudio de la extrema derecha. A continuación, su artículo propone presentar un análisis inicial sobre esta ideología política en Brasil, enfocando en la construcción del perfil sociocultural e ideológico del gobierno del actual presidente Jair Bolsonaro. Su intención es analizar la forma en que el gobierno brasileño redefine y considera las identidades étnico-raciales y cómo construye su propia identidad al oponerse a ellas. Al centrarse en la problemática de la alteridad indígena, se propone observar cómo es resignificada en el proyecto de Bolsonaro. La autora destaca el encuentro entre la extrema derecha y la alteridad en el contexto brasileño lo que le permite verificar los valores y significados que el actual gobierno da a la pluralidad cultural, los imaginarios y representaciones sociales que construye. En un recuento minucioso de discursos, políticas públicas y polarizaciones, la autora procura dar cuenta sobre cómo se presenta y qué proyecto de sociedad defiende este gobierno de extrema derecha.

El artículo de Eduardo Restrepo Hacer antropología hoy desde América Latina: apuntes en torno a la reinvención de nuestras antropologías, reflexiona sobre tres campos problemáticos y transformaciones que dibujan las antropologías latinoamericanas que lo llevan a pensar en la necesidad de reinventar nuestra disciplina, a saber, la creciente digitalización de nuestras experiencias y existencias, los procesos de derechización, los entrampamientos burocráticos y la precarización laboral. Coincidiendo con Myriam Jimeno y otras.os colegas, afirma que las Américas Latinas asumidas como un horizonte ético-político articulado en las márgenes de una geopolítica del conocimiento y de un sistema mundo de la antropología. Por ello nuestras antropologías 
implican un posicionamiento, antes que una posición de sujeto dada por una ubicación geográfica; suponen una articulación ético-política a partir de sus lugarizaciones. Es ese posicionamiento o articulación lo que define el locus de enunciación en nuestras antropologías. En concordancia con Ribeiro (2018) y García Canclini (2019) afirma que con el denso entramado de las tecnologías digitales se ha profundizado la acumulación de capital, se ha potenciado de disimiles maneras las articulaciones del mercado, y afinado los dispositivos de control y gobierno. Nuestras sensibilidades e inteligibilidades se producen y cristalizan desde algoritmos que nos constituyen como mercancía, que definen burbujas de odio, afinan las capacidades de control y marcan nuestras subjetividades, tanto como posibilidades de accesos e interacciones (García Canclini, 2019). Le preocupan los sistemas de evaluación de la ciencia, la burocratización e individualización del trabajo académico que debieran ponerse en entredicho. Al igual que Rebecca Lemos Igreja, coloca como el segundo reto los giros a la derecha y sus implicaciones para la vida cotidiana, la ciencia y los derechos humanos que pueden ser etnografiados para comprender la compleja relación y explicación de los autoritarismos que se han instaurado a través del voto popular. Un tercer eje problemático lo constituye las ascendentes tendencias de precarización del mercado laboral y jerarquización en las instituciones académicas que desde su perspectiva son una expresión de la derechización que recorre nuestra América Latina. Son temas que nos tocan como académicas.os, antropólogas. os y ciudadanas.os que deben ser visibilizados y analizados.

Desde una postura crítica al multiculturalismo y sus políticas de reconocimiento de derechos culturales, y en este caso centrada en los derechos políticos electorales, Laura Valladares en su artículo Una mirada antropológica a la usurpación de la identidad indígena en las elecciones de 2018 y 2021 en México, analiza las cuotas afirmativas que en materia electoral para pueblos indígenas se han normado, específicamente para el acceso a curules en la Cámara de Diputados Federal en dos legislaturas (2018-2021) y la entrante (2021-2023). La autora debate acerca de los avances y retos que enfrenta la democracia representativa para los pueblos indígenas. Considera que hacer una etnografía de los procesos electorales se constituye como un observatorio que nos permite un acercamiento sobre el papel que tiene la problemática indígena en el marco del Estado nacional mexicano. Otra de las dimensiones que explora es la resistencia de los partidos políticos nacionales para incorporar candidatos indígenas, misma que puede entenderse por la existencia de un racismo prevaleciente en los actores políticos, quienes consideran que los indígenas tienen poca preparación y poco que aportar a los grandes temas de interés nacional. Si bien reconoce que las acciones afirmativas son un avance positivo en el reconocimiento de los derechos indígenas, con miras a construir una democracia participativa en donde la pluriculturalidad y la equidad de género sean parte del ejercicio democrático, son todavía un proyecto a largo plazo. Laura Valladares muestra como en los dos procesos electorales estudiados se han presentado casos inéditos de suplantación de la identidad indígena y que al igual que la conquista de la normatividad que llevó a la paridad de género en los procesos electorales, la participación indígena en el poder legislativo se ha conquistado a golpe de sentencias, con las que se busca resarcir la sub representación indígena, sin embargo, prevalecen las pugnas por el control del poder nacional, las luchas faccionales y la corrupción de los actores y los partidos políticos. En un escenario de enorme vaciamiento de los debates políticos e ideológicos, de la banalización de la política, de un pragmatismo en donde los actores políticos van de un partido a otro sin importar las plataformas políticas y los principios ideológicos, en que prevalece una lucha por acceder a la máxima tribuna del poder legislativo, la participación de las y los indígenas es prácticamente marginal.

En esta línea reflexiva sobre el último proceso electoral en México, Cristina Oehmichen en su artículo La Antropología frente a la racialización de los procesos político-electorales en México analiza, desde una perspectiva antropológica, el racismo y los procesos de racialización y polarización social en México observados en las prácticas discursivas y publicidad electoral llevadas a cabo con motivo de los comicios de junio de 2021. A partir de la propaganda electoral, la autora da cuenta del uso de estereotipos racistas y clasistas que fueron ampliamente difundidos a lo largo de la campaña electoral. Cristina Oehmichen afirma que estos discursos 
forman parte de determinados marcos de referencia primarios que funcionan como esquemas de interpretación de la realidad social y son puestos a prueba en la contienda política. Dichos marcos constituyen esquemas de percepción e interpretación que permiten a los sujetos reafirmar su propia identidad, distinguirse y orientar su actuar más allá de la contienda electoral.

Los dos siguientes artículos colocan sus reflexiones en el campo de novedosas metodologías antropológicas, que abrevan y se enriquecen de modelos teóricos y metodológicos de otras disciplinas, como son la educación popular o la investigación acción, entre los más significativos. Procuran demostrar la forma en que las etnográficas y la construcción de teorías antropológicas se enriquecen del diálogo con otras tradiciones disciplinarias y al colaborar con los actores con los que se trabaja y se hace investigación. El primero de los artículos fue elaborado por Joanne Rappaport, What was Research for the Peasants of Córdoba? Participation in the Early Years of Participatory Action Research, quien, desde su larga experiencia de investigación participativa y colaborativa en Colombia, recupera una dimensión poco trabajada y muy sugerente, que se refiere a los significados y experiencias de vida para las.os investigadoras.es comunitarias.os cuando colaboran en una investigación sobre sus propias realidades. De acuerdo con el estudio de Rappaport, estas.os investigadoras.es suelen adoptar formas muy diferentes a las de las.os académicas.os. Ellas.os pueden utilizar métodos que son explícitamente intuitivos y pueden apartarse de los enfoques que implican la recopilación y sistematización rigurosa de datos. En este sentido, este artículo explora lo que la investigación pudo haber significado para los campesinos caribeños de principios de la década de 1970 con quienes el sociólogo colombiano Orlando Fals Borda desarrolló su enfoque de lo que hoy se llama investigación-acción participativa. En particular, se centra en las notas de campo de Alfonso Salgado Martínez, líder de la Asociación Nacional de Usuarios CampesinosLínea Sincelejo (ANUC, Asociación Nacional de Usuarios Campesinos-Línea Sincelejo), yuxtaponiéndolas a su trabajo publicado, ambos leídos en comparación a las notas y escritos del propio Fals Borda. La autora muestra que se trata de un discurso basado en iconos y sentimientos en lugar de los datos, a pesar de que se nutre de la investigación. Para comprender tal afirmación es necesario replantear la investigación como la asimilación a través de la acción política de todas las historias y experiencias a las que Salgado estaba al tanto sobre su vida y no en un mismo sentido que la emprendemos como académicas.os.

Considera Rappaport que quizás solo cuando reconozcamos lo que la investigación significa para otros, podremos avanzar en la investigación colaborativa. A partir de las 14 preguntas documentadas por Alfonso Salgado, y estudiadas por la autora, muestra que los investigadores campesinos encontraron sus propios medios a través de los cuales se apropiaban de los que aprendieron en los cursillos, de lo que escucharon cuando visitaron las comunidades, y de lo que recordaban de la experiencia personal. Concluye señalando que la experiencia de Fals Borda y la Fundación del Caribe que aborda en su artículo no nos brinda todas las respuestas, pero ciertamente nos lleva hacia las preguntas que debemos hacernos mientras perseguimos estrategias de investigación colaborativa.

El siguiente artículo es de la autoría de la antropóloga guatemalteca Marta Casaus, que bajo el título de El diagnóstico del racismo y la discriminación en Guatemala: Metodología cualitativa y participativa para la elaboración de una política pública, nos presenta un recuento detallado de la implementación de una metodología interdisciplinaria y participativa, basada en un modelo de investigación-acción participativa y en la construcción colectiva de conceptos que permitieran valorar el racismo y la discriminación y sus efectos económicos y sociales en la población discriminada en Guatemala. Se trata de un trabajo innovador porque los resultados de esta investigación tenían un objetivo práctico muy relevante: que sirviera de insumo científico para la creación de una política pública que paliara la discriminación étnica y de género y que sentara las bases de un Estado plural que favoreciera a toda la ciudadanía y promoviera la igualdad de trato y de oportunidades. La investigación se centró en cinco líneas: 1)Los costos económicos de la discriminación y el desigual acceso a los bienes y servicios del Estado; 2)La perspectiva jurídico-constitucional y de jurisprudencia en materia de igualdad compensatoria 
y de discriminación positiva; 3)El mapa conceptual y las prácticas sociales discriminatorias sobre el racismo y la discriminación que nos permitieran crear términos, conceptos y políticas consensuadas; 4)Las prácticas discursivas y los estereotipos en los medios de comunicación y las redes sociales; 5)Las historias de vida y la voz del sujeto discriminado y racializado por etnia y género.

Los resultados de esta investigación fueron muy esclarecedores y el gobierno guatemalteco se comprometió a establecer políticas públicas y acciones que contribuyeran a desterrar el racismo, sin embargo, las condiciones políticas y financieras del país no lograron dar continuidad a lo pactado. Es una experiencia muy sugerente de un trabajo académico serio, profundo y comprometido que se construye específicamente y a solicitud del gobierno de esa nación en donde el racismo tiene profundas huellas y consecuencias sociales, políticas y económica. Se trata de una investigación que habla en un lenguaje que es comprensible a los actores políticos, mostrando los costos que tiene para el Estado perpetuar un modelo basado en la discriminación y la desigualdad. Es un trabajo que da cuenta ampliamente sobre la importancia de los saberes de la antropología para la resolución de uno de los grandes problemas que aquejan a nuestras sociedades.

Un tercer conjunto de artículos está dedicado a reflexionar sobre y desde las antropologías nacionales, desde las cuales se da cuenta acerca de la construcción de las antropologías en Chile, Centroamérica y Ecuador. El primer texto fue preparado por Francisca Márquez, a partir de la experiencia chilena, y nos ofrece un recorrido muy estimulante sobre los avatares de la antropología chilena, bajo el título de Antropologías en el extremo sur. Del silenciamiento al estallido. Se construye a partir de un recorrido por la genealogía de los saberes y las.os investigadoras.es destacadas.os de la disciplina, revisando huellas, sus preguntas y la vigencia de sus planteamientos en el presente. Fue organizado en torno a algunos de los principales nudos temáticos que han acompañado a la disciplina en Chile: memoria y derechos subalternos; gente de la tierra, silenciamiento y guerra; feminismo y disidencias; heterotopías en el patrimonio; ecología. La autora concluye su artículo con los desafíos para la construcción y consolidación de una antropología crítica y comprometida con los violentos e incesantes procesos de transformación de las culturas de su país.

Por su parte Marisa Ruiz nos presenta un texto que nos acerca a la influencia del marxismo y la perspectiva gramsciana en la conformación de una antropología feminista en una región conformada por el fronterizo estado mexicano de Chiapas y Centroamérica, bajo el título de Marxistas y gramscianas: referentes de la antropología feminista en Chiapas y Centroamérica (1970-1992). La autora muestra que la antropología feminista ha conformado un campo de conocimiento dentro de la disciplina desde una perspectiva de género. Partiendo de la escasez de trabajos sobre el desarrollo de las antropologías feministas locales, se concentra en recuperar algunos referentes importantes de la antropología feminista chiapaneca y centroamericana, específicamente en el desarrollo de lo que considera como un subcampo temático entre los años setenta y noventa (S. XX), que tiene la particularidad de ser un periodo histórico marcado por la convulsión política, la represión y las políticas de contrainsurgencia en Centroamérica, lo que afectó fuertemente a estudiantes y profesoras de la época. Se trata de un trabajo relevante porque recupera los aportes de antropólogas notables como Alaíde Foppa, Stella Quan, Walda Barrios y Marta Casaus y analiza no solamente su quehacer antropológico y sociocientífico sobre las críticas feministas marxistas y socialistas a las nociones tradicionales de clase, las influencias del pensamiento gramscicano, los cuestionamientos anticoloniales de la época que marcaron su pensamiento, sino también sus experiencias políticas en movimientos sociales y revolucionarios, así como algunas de sus vivencias personales. Podemos ubicarlo como parte de las reflexiones de una antropología feminista que se ha ampliado y fortalecido en América Latina, especialmente en contextos de enorme violencia y conflictos políticos, en la cual las voces, propuestas y aportes de las antropólogas feministas en la región centroamericana y chiapaneca son poco conocidas. 
Mientras que Carolina Páez y Fernando García en su artículo 50 años de profesionalización de la antropología ecuatoriana: temas, derroteros y perspectivas nos presentan un recuento de las temáticas y grandes preocupaciones de la antropología ecuatoriana, principalmente, a partir de la producción de las tesis elaboradas por los estudiantes de antropología en un periodo que va de 1977-2019. La autora y el autor profundizan su análisis recurriendo a la realización, a la realización de grupos focales con miembros de las primeras generaciones de antropólogas.os formadas.os en el país. Los temas principales giraron en torno a la experiencia universitaria y a la experiencia laboral. La propuesta se basa en el hecho que, si bien en las investigaciones fruto del proceso formativo se puede observar la multifacética "naturaleza" de la antropología, existen unos núcleos temáticos bien definidos tanto en la investigación formativa como una particular característica del quehacer antropológico. El eje que atraviesa estos núcleos temáticos es la pregunta por la identidad en sus diversas manifestaciones. Así, buscan hacer un recuento de la institucionalización de la formación antropológica y su diversificación; presentar los temas de las investigaciones en los últimos años y, finalmente, discutir sus resultados en relación con los retos que enfrentan las antropologías latinoamericanas y la ecuatoriana en específico.

Cierra este dossier con dos artículos que, desde una perspectiva de la Antropología abordan el campo del derecho y de la justicia. La antropología jurídica y la antropología del derecho se han destacado como uno de los campos más promisorios de la disciplina en América Latina en los últimos años, especialmente por su activismo en la defensa de los derechos, por el acceso a la justicia y acerca de la justicia social para las poblaciones más vulnerables en el continente. Es importante resaltar que ambos artículos exponen como particularidad la experiencia del trabajo antropológico realizado a través de la construcción de redes latinoamericanas sostenidas por proyectos de investigación colaborativos que buscan promover intercambios de conocimiento y de datos. Retratan de esta manera la importancia de promover un diálogo fructífero entre las antropologías latinoamericanas.

El primer artículo, Jean Muteba Rahier The Observatory ofJustice for Afrodescendants in Latin America (OJALA) as an initiative of engaged anthropology for the promotion and defense of human rights, presenta la experiencia de una iniciativa de antropología comprometida que originó el Observatorio de Justicia para Afrodescendientes en América Latina (OJALA), ubicado en el Centro Kimberly Green para América Latina y el Caribe (KG-LACC) en Florida International University (FIU). El observatorio se ocupa de uno de los grandes temas de la antropología que se vincula al estudio de la población afrodescendiente del continente americano, su situación social, económica, sus procesos reivindicativos y las especificidades de su expresiones culturales e identitarias. Su foco está, especialmente, en cómo el estado multiculturalista latinoamericano "funciona" en las relaciones concretas que teje con ciudadanos afrodescendientes y lo que hace o no hace el Sistema de justicia estatal en la defensa de sus derechos. Su análisis está construido con base en estudios etnográficos comparativos sobre litigios específicos presentados por afrodescendientes en los sistemas de justicia de varios países de América Latina.

El artículo que cierra este número de la revista Vibrant, trata acerca de la experiencia de la construcción de redes latinoamericanas de antropología con la contribución de Guadalupe Irene Juárez Ortiz y Welliton Caixeta Maciel, sobre El Foro Latinoamericano de Antropología del Derecho (FLAD): Diálogos, conexiones y retos entre México, Brasil y Argentina. El artículo se centra en describir y reflexionar, con base en un enfoque histórico y etnográfico, sobre la antropología jurídica y del derecho latinoamericana desde el trabajo realizado por el FLAD. La finalidad es recuperar la memoria y objetivos de sus dos precursores, como un foro académico-científico por medio del cual se pueda ubicar y vincular a los grupos, laboratorios y centros de investigaciones, sí como a las.os investigadoras.es y estudiantes cuyo trabajo están centrados en la reflexión antropológica del Derecho a partir de la etnografía de las instituciones jurídicas y burocracias estatales; sobre sus múltiples manifestaciones y 
tensiones, en México, en Brasily en Argentina. El artículo presenta la experiencia innovadora de una iniciativa que parte de posgraduados latinoamericanos y que alcanza una expresión regional importante al estimular el diálogo sobre campos temáticos nacionales específicos, abordando sus convergencias y diferencias.

Aunque este dossier no tenga la pretensión de abarcar la multiplicidad de las antropologías latinoamericanas, pues deja de fuera antropólogas.os importantes, así como varios países en que la disciplina se ha desarrollado ampliamente, busca destacar la diversidad de estudios, temas, dilemas y desafíos del quehacer antropológico en la contemporaneidad en la región. El deseo es que pueda contribuir para el debate sobre la disciplina a lo cual tantas.os colegas en toda la región se han dedicado. De manera especial, esperamos que contribuya con el fomento del diálogo entre las distintas antropologías latinoamericanas y, particularmente, con la antropología brasileña.

Recibido: 13/09/2021

Acepto: 14/09/2021

\section{Referencias Bibliográficas}

BARTRA, Roger. 2021. Regreso a la Jaula. El fracaso de López Obrador. México: Editorial Debate

GARCÍA CANCLINI, Néstor. 2019. Ciudadanos reemplazados por algoritmos. México: Edicciones: CALAS Maria Sibylla Merian Center.

JIMENO, Myriam; ARIAS, David. 2011. "La enseñanza de antropólogos en Colombia: Una antropología ciudadana”. Alteridades, 21(41): 27-44.

LEYVA, Xochitl et al. 2015. Prácticas otras de conocimiento(s). Entre crisis, entre guerras. México, Colección Conocimientos y Prácticas Políticas. Buenos Aires:: CLACSO; Chiapas: Cooperativa Editorial Retos; Lima: Programa Democracia y Transformación Global (PDTG); Copenhague: Grupo Internacional de Trabajo sobre Asuntos Indígenas (IWGIA); La Habana: Talleres Paradigmas Emancipatorios-Galfisa; Coimbra: Proyecto Alice - Espejos Extraños, Lecciones Insospechadas; Guadalajara: Taller Editorial La Casa del Mago, 2018.

LINS RIBEIRO, Gustavo. 2018. "Giro global a la derecha y la relevancia de la antropología". Encartes, 1(1): 5-26. https://doi.org/10.29340/en.v1n1.8 . Recuperado el 10 septiembre 2021.

Laura R Valladares

https://orcid.org/0000-0002-7577-6300

Email: lauravalladares.delacruz@gmail.com

Rebecca Lemos Igreja

https://orcid.org/00oo-0002-9533-2985

Email: rebeccaigreja@unb.br 